\title{
Idiopathic Hypersomnia and Depression, the Challenge for Clinicians and Researchers
}

\section{Karolína Galušková, Karel Šonka}

Department of Neurology and Center for Clinical Neuroscience, First Faculty of Medicine, Charles University and General University Hospital in Prague, Prague, Czech Republic

Received September 19, 2020; Accepted August 5, 2021.

Key words: Depressive symptoms - Excessive daytime sleepiness - Hypersomnia associated with a psychiatric disorder - Idiopathic hypersomnia - Mood disorder

Abstract: The review deals with idiopathic hypersomnia, focusing mostly on the research findings about the presence, onset and severity of excessive daytime sleepiness and depressive symptoms in patients with idiopathic hypersomnia.

This study was supported by Ministry of Health of the Czech Republic, grant No. NU20-04-00088.

Mailing Address: Mgr. Karolína Galušková, Sleep and Wake Disorders Center, Department of Neurology and Center for Clinical Neuroscience, First Faculty of Medicine, Charles University and General University Hospital in Prague, Kateřinská 30, 12000 Prague 2, Czech Republic; Phone: +420 224965 550; e-mail: karolinagaluskova.kg@gmail.com 


\section{Introduction}

Idiopathic hypersomnia $(\mathrm{IH})$ was identified and named in the $20^{\text {th }}$ century by renowned Czechoslovak neurologist, neurophysiologist and sleep researcher Bedrich Roth.

In 1956 the first description of $\mathrm{IH}$ appeared in Roth's article Sleep drunkenness and sleep paralysis in Czechoslovak Neurology. In 1976 Roth published the comprehensive description of the disorder. In the same publication the term idiopathic hypersomnia was used first and gained a general acceptance. We have come a long way since Roth's first work. However, there is still much work to be done. There is an absence of a sufficiently strong $\mathrm{IH}$ biomarker, poor epidemiological characterization, little knowledge of pathophysiology and no registered treatment.

The aim of this paper is to present the main findings concerning $\mathrm{IH}$ and to summarize the current and past information on depressive symptoms in $\mathrm{IH}$. Because there is a discrepancy in the literature about the relationship between $\mathrm{IH}$ and depressive symptoms we reopen the existing, important question of whether the hypersomnolence in $\mathrm{IH}$ comes first or whether depressive symptoms (or mood disorder) are the first to appear. We speculate about the connection of pathophysiological aspects of both $\mathrm{IH}$ and mood disorders. This paper also discusses the problematic distinguishing among $\mathrm{H}$, hypersomnia associated with a psychiatric disorder and mood disorder.

\section{Idiopathic hypersomnia - Current classification, diagnosis, differential diagnosis, and symptoms}

Two main diagnostic manuals are commonly used to assist in diagnosing sleep disorders: the third edition of the International Classification of Sleep Disorders (ICSD-3) (American Academy of Sleep Medicine, 2014) and the fifth edition of the Diagnostic and Statistical Manual of Mental Disorders (DSM-5) (American Psychiatric Association, 2013).

The ICSD-3 uses the term idiopathic hypersomnia (American Academy of Sleep Medicine, 2014) while according to the DSM-5 the disorder is called hypersomnia (American Psychiatric Association, 2013).

The ICSD-3 classifies IH as a central disorder of hypersomnolence $(\mathrm{CDH})$. For the sake of completeness, it should be pointed out that the term hypersomnolence is used to name the symptom of excessive sleepiness, whereas hypersomnia refers to specific disorders, such as IH (American Academy of Sleep Medicine, 2014).

Hypersomnolence disorders may be divided into those that are primary, meaning they are not caused by another condition and disrupted night sleep namely narcolepsy type 1 (NT1) with cataplexy, narcolepsy type 2 (NT2) without cataplexy, $\mathrm{IH}$ and Kleine-Levin syndrome and those that are secondary, in which sleepiness is believed to be caused by the other disorders, medication, or short habitual sleep time. 
Patients with IH suffer from periods of an irrepressible need for sleep or daytime lapses into sleep occurring for at least three months despite undisturbed nocturnal sleep of good quality. These periods occur according to the ICSD-3 daily (American Academy of Sleep Medicine, 2014), and according to DSM-5 at least 3 times a week (American Psychiatric Association, 2013). IH does not involve cataplexy. Sleep-onset REM period (SOREM) may occur on Multiple Sleep Latency Test (MSLT) and preceding night polysomnography (PSG) together less than twice. Objective evidence of hypersomnolence in $\mathrm{IH}$ must be demonstrated by MSLT showing the mean sleep latency (MSL) of $\leq 8$ minutes, and/or by a total sleep time of $\geq 660$ minutes/24 hours recorded by PSG or by wrist actigraphy and a sleep diary. In addition to that, sleep efficiency (SE) on PSG is usually above $90 \%$ in IH patients while self-reported quality of sleep may be poor. Patients typically do not easily wake up and often use special procedures or need someone who wakes them up (American Academy of Sleep Medicine, 2014).

Compared to narcolepsy, sleepiness in IH does not have an imperative character (Vernet et al., 2010). Hypersomnolence may fluctuate in severity and occurs typically in monotonous situations that require a low activation (while attending lectures, reading, watching TV), but may appear in situations demanding more attention (meeting at work or social event) as well. Naps are generally long, often longer than 60 minutes and described as unrefreshing by $46 \%$ to $78 \%$ of patients (American Academy of Sleep Medicine, 2014). The self-administered questionnaire Epworth Sleepiness Scale (ESS) is commonly used to measure the subjective excessive daytime sleepiness (EDS) (Johns, 1991).

Roth distinguished monosymptomatic and polysymptomatic form of $\mathrm{IH}$ (Roth, 1976). The monosymptomatic form manifests itself by EDS, while polysymptomatic form is characterized by EDS, prolonged night sleep usually of 12-18 hours duration and signs of sleep inertia historically known as sleep drunkenness (SD) upon awakening. Sleep drunkenness consists of prolonged and difficult period between awakening until the full wakefulness associated with repeated returns to sleep, disorientation, irritability and poor coordination (American Academy of Sleep Medicine, 2014).

Similarly to Roth (1976), the previous edition of the International Classification of Sleep Disorders (ICSD-2) distinguished two IH phenotypes as independent nosological units: $\mathrm{IH}$ with long sleep time (LST) (i.e. > 10 hours for the main sleep period) and $\mathrm{IH}$ without long sleep time (American Academy of Sleep Medicine, 2005).

The ICSD-3 merged two IH forms into one unit because of the insufficient diversity of these forms (Vernet and Arnulf, 2009; American Academy of Sleep Medicine, 2014). However, it has been suggested that IH with LST may be a juvenile form of the same disorder, later evolving towards less sleep in night.

In favour of this hypothesis is that total sleep time during night-time and during 24 hours slightly decreased with increasing age at diagnosis time (Vernet and Arnulf, 
2009). A longitudinal follow-up of IH patients would be helpful to support this hypothesis.

Monosymptomatic form of $\mathrm{IH}$ is not very different from NT2. The only distinction between them is the presence of two or more SOREM periods on the MSLT or preceding PSG in NT2. Moreover, the SOREM occurrence is not stable in IH and NT2 (Trotti et al., 2013; Šonka et al., 2014; Lopez et al., 2017b; Ruoff et al., 2018). Thus $\mathrm{IH}$ (especially its monosymptomatic form) and NT2 may be the same condition (Lammers et al., 2020).

The differential diagnosis of $\mathrm{IH}$ consists primarily in the exclusion of chronic sleep deprivation, then other CDH, especially NT1 and NT2. EDS due to other sleep disorders, particularly obstructive sleep apnea (OSA) and periodic limb movement disorder (PLMD) must be also ruled out.

Mental illnesses, medication use, and substance abuse or head injury must be also considered and excluded (American Academy of Sleep Medicine, 2014). EDS must not be better explained by internal, autoimmune (Braga et al., 2016) and neurological disorders such as neurodegenerative diseases (Arnulf, 2005; Moreno-Lopez et al., 2011) or inflammatory diseases, particularly neuroinfections (Pardasani et al., 2008; Krbkova et al., 2015).

All the criteria for $\mathrm{IH}$ that must be met are shown in Table 1.

\section{Epidemiology of IH and disease course}

$\mathrm{IH}$ is a rare disorder, and its robust epidemiological studies are lacking. There is not valid data, but IH appears to be one-tenth to one-half as common as narcolepsy (Billiard, 1996; Bassetti and Aldrich, 1997; Anderson et al., 2007).

The age of symptom onset varies, but usually in adolescence or early adulthood (Anderson et al., 2007; American Academy of Sleep Medicine, 2014) although diagnosis is commonly delayed (Bassetti and Aldrich, 1997; Anderson et al., 2007).

For example, in the study of $77 \mathrm{IH}$ patients, their mean age was 17 years at symptom onset and their mean age was 30 years at diagnosis (Anderson et al., 2007). Clinical experience suggests a higher incidence in women than in men (Roth, 1976; Bassetti and Aldrich, 1997; Anderson et al., 2007). The course of $\mathrm{IH}$ is not well studied, but once established, the disorder is generally stable in severity and long lasting (American Academy of Sleep Medicine, 2014), although a spontaneous remission has been reported in some IH patients (Bassetti and Aldrich, 1997; Anderson et al., 2007; Kim et al., 2016). However, factors that predict such remission remain unknown.

\section{Etiology of IH}

The term idiopathic is used to describe a disorder with no clear cause. One hypothesis assumes altered $\mathrm{GABA}_{\mathrm{A}}$ receptor inhibitory signalling in the etiology of IH (Rye et al., 2012). Other hypotheses consider the autoimmune mechanism (Barateau et al., 2017a; Lippert et al., 2019), the impact of infectious mononucleosis 


\section{Table 1 - Diagnostic criteria of idiopathic hypersomnia according to ICSD-3 (American Academy of Sleep Medicine, 2014)}

\section{Criteria A-F must be met:}

A) The patient has daily periods of irrepressible need to sleep or daytime lapses into sleep occurring for at least three months.

B) Cataplexy is absent.

C) An MSLT performed according to standard techniques shows fewer than two sleep onset REM periods or no sleep onset REM periods if the REM latency on the preceding polysomnogram was less than or equal to 15 minutes.

D) The presence of at least one of the following:

1. The MSLT shows a mean sleep latency of $\leq 8$ minutes.

2. Total 24 -hour sleep time is $\geq 660$ minutes (typically $12-14$ hours) on 24 -hour polysomnographic monitoring (performed after correction of chronic sleep deprivation), or by wrist actigraphy in association with a sleep log (averaged over at least seven days with unrestricted sleep).

E) Insufficient sleep syndrome is ruled out (if deemed necessary, by lack of improvement of sleepiness after an adequate trial of increased nocturnal time in bed, preferably confirmed by at least a week of wrist actigraphy).

F) The hypersomnolence and/or MSLT findings are not better explained by another sleep disorder, other medical or psychiatric disorder, or use of drugs or medications.

ICSD-3 - third edition of the International Classification of Sleep Disorders; MSLT - Multiple Sleep Latency Test

(Sforza et al., 2018) or abnormality of circadian rhythm in the pathophysiology of $\mathrm{IH}$ (Vernet and Arnulf, 2009; Lippert et al., 2014; Materna et al., 2018).

\section{Depressive symptoms in IH}

Up to now no questionnaire has been validated to assess the presence and the severity of depressive symptoms in patients with CDH (Lopez et al., 2017a). Screening questionnaires for depressive symptoms, such as the Beck Depression Inventory (BDI) is largely administrated in patients with $\mathrm{CDH}$ (Beck et al., 1961).

The Hamilton Depression Rating scale (HDRS), the Montgomery Asberg Rating scale (MADRS) or the Hospital anxiety and depression scale (HADS) are other commonly used instruments to quantify the severity of depressive symptoms (Hamilton, 1960; Montgomery and Asberg, 1979; Zigmond and Snaith, 1983).

We agree with Lopez and colleagues (2017a) that a structured (standardized) or semi-structured interview remain essential in the assessment of depressive symptoms in $\mathrm{IH}$ and hypersomnia associated with a psychiatric disorders.

Several studies have pointed to the presence of depressive symptoms in $\mathrm{IH}$ patients (Roth et al., 1971; Anderson et al., 2007; Vernet et al., 2010; Kim et al., 2016; Lee et al., 2017; Neikrug et al., 2017; Dauvilliers et al., 2019; Pascoe et al., 2019; Pomares et al., 2019). There is no consensus in the literature whether the 
frequency of depressive symptoms is higher in $\mathrm{IH}$ patients or in narcoleptics. For example, in a study of Kim and colleagues (2016), the frequency of depressive symptoms was significantly higher in IH than in both NT1 and NT2.

In 1971 Roth and colleagues reported symptoms of recurrent depression in $\mathrm{IH}$ patients. Patients suffered from symptoms called by the authors as neurotic such as difficulties in concentration, emotional lability, and anxiety (Roth et al., 1971). Such findings are congruent with following investigation of Neikrug and colleagues (2017) because the most typical symptoms in $\mathrm{IH}$ patients were difficulty with concentration, irritability, loss of interest and anxiety. Recently, Trotti and colleagues (2020) reported in a web based questionnaire study symptoms such as brain fog defined as being unable to think clearly or concentrate at any time throughout the day and poor memory as a daily problem in most $\mathrm{IH}$ respondents.

However, there is no evidence on whether these additional symptoms are specifically related to $\mathrm{IH}$ either as a primary symptom more or less prominent or the consequence.

The studies with $\mathrm{IH}$ participants frequently lack healthy controls, as $\mathrm{IH}$ is usually compared with narcolepsy and the sample size of most of these studies is relatively small (Bassetti and Aldrich, 1997; Vernet and Arnulf, 2009). The lack of knowledge in this area may be caused by excluding $\mathrm{IH}$ patients from the study in case they have any coexistent psychiatric symptoms. Moreover, there is so little information about pediatric $\mathrm{IH}$ patients in the connection with depressive symptoms and thus $\mathrm{IH}$ in childhood will not be included in this review. Furthermore, according to our information, up to now no study has longitudinally examined depressive symptoms and their severity in $\mathrm{IH}$ patients.

\section{IH, hypersomnia associated with a mood disorder and mood disorder - A diagnostic challenge}

According to DSM-5, the hypersomnia is described in the association with mood disorders such as bipolar disorder (in depressive stage) or seasonal affective disorder. Patients with hypersomnia may suffer from depressive symptoms and may fulfil the diagnostic criteria for a mood disorder. The DSM-5 allows hypersomnia to be diagnosed independently from the presence of a current or past mood disorder because depressive symptoms may be connected with psychosocial consequences of EDS (American Psychiatric Association, 2013).

Contrary to the ICSD-3, the diagnosis of $\mathrm{IH}$ requires ruling out mood disorders. Patients with a psychiatric condition, most typically depression, should be diagnosed with hypersomnia associated with a psychiatric disorder. Nevertheless, the presence of depressive symptoms (not fulfilling the diagnostic criteria of the depression disease) does not exclude the diagnosis of $\mathrm{IH}$ according to ICSD-3 (American Academy of Sleep Medicine, 2014).

Currently the differential diagnosis to distinguish $\mathrm{IH}$ from a mood disorder with EDS subjective symptoms relies heavily on the MSLT results. It is widely accepted, 
that patients with a mood disorder do not have a short mean sleep latency of $\leq 8$ minutes (Billiard and Dauvilliers, 2001; American Academy of Sleep Medicine, 2014). The complaint of EDS and prolonged sleep may be similar in patients with $\mathrm{IH}$, but in mood disorders the degree of EDS is not stable over time. The SE on PSG in $\mathrm{IH}$ patients is usually above $90 \%$ while in mood disorders the night sleep tends to be of poor quality (Billiard and Dauvilliers, 2001).

The differential diagnosis relies on MSLT results to distinguish $I \mathrm{H}$ from hypersomnia associated with psychiatric disorder as well. The study comparing the night-time sleep duration between the group of patients with a hypersomnia associated with a psychiatric disorder and the group of $\mathrm{IH}$ revealed that $14 \%$ of psychiatric hypersomniacs slept over 9 hours at night and $36.1 \%$ of psychiatric hypersomniacs had a reduced MSL (Billiard et al., 1994). Such findings are congruent with a recent meta-analysis of Plante (2017).

Lammers and colleagues (2020) disagree with a current ICSD-3 classification and suggest entities such as hypersomnia associated with a psychiatric disorder, hypersomnia due to medical disorder, hypersomnia due to substance abuse should be discontinued because in most cases it is not presently known if the relationship is truly causal or simply co-morbid (Barateau et al., 2017b; Plante, 2017). Instead, medical disorders and psychiatric disorders including substance abuse should be considered and listed as possible co-morbidities. This would be in line with decisions made by ICSD-3 to allow a diagnosis of insomnia independently from the presence of a psychiatric disorder, medical condition, drug or substance intake (Lammers et al., 2020).

It would be also in line with DSM-5 allowing a diagnosis of hypersomnia independently from the presence of a current or past psychiatric disorder (American Psychiatric Association, 2013).

\section{EDS in mood disorders}

The evidence suggests the frequency of EDS is high in mental health disorders and particularly in mood disorders (Detre et al., 1972; Akiskal and Benazzi, 2005; Kaplan and Harvey, 2009; Lopez et al., 2017a; Plante et al., 2017).

EDS in major depressive disorder (MDD) significantly varies across age, ranging from $8.9 \%$ in childhood ( $<13$ years) to a high rate of $75.8 \%$ in young adulthood. EDS also varies across gender, but appears to be more prevalent in females than in males (Kaplan and Harvey, 2009). The large frequency range likely reflects nonuniform definitions of EDS and assessing the EDS by subjective questionnaires only, or simple questions on sleep duration.

According to Dauvilliers and colleagues (2013), EDS in mood disorders is a subjective sleep complaint rather than an objective finding. Dysthymic patients did not show abnormalities on the MSLT, neither on the PSG compared to a group of $\mathrm{IH}$ patients. However, dysthymic patients showed an excess of sleep stage NREM 1 and a decrease of stages NREM 3 and 4, which could be related to their complaint of 
EDS (Dolenc et al., 1996). Importantly, since the update of the classification of sleep stages (the American Academy of Sleep Medicine - AASM) in 2007, NREM 3 and NREM 4 are combined as stage N3 (Iber et al., 2007).

It seems that these patients tended to remain in bed without an objective evidence for increased sleep time. This behaviour is known as clinophilia (American Academy of Sleep Medicine, 2014).

\section{The chronology of onset of depression and EDS}

The chronology of onset of mood disorders and EDS could help to clarify the relationship between these two conditions. In the case of suspicion of $\mathrm{IH}$ and the presence of depressive symptoms, it is relevant to study the date of onset of each symptom, the evolution of EDS depending on mood and the effect of the treatment of depressive symptoms on EDS and vice versa.

There is still a lack of knowledge about $\mathrm{IH}$ in a clinical practice, it is possible that due to general knowledge about mood disorders, some patients with $\mathrm{IH}$ are misdiagnosed and treated as depression and diagnosis of $\mathrm{IH}$ is thus delayed.

EDS in mood disorders is commonly considered to be a consequence of the disorder, in the line with monoamine activity disturbances (especially catecholamines, dopamine or serotonin) (Lopez et al., 2017a; Ogawa et al., 2018). The meta-analysis of Ogawa and colleagues (2018) revealed that homovanillic acid levels (often used as an indicator of dopaminergic activity) were decreased in the cerebro-spinal fluid of patients with a mood disorder (Lambert et al., 1993; Ogawa et al., 2018).

The study of Roth (1956) shown that depressive symptoms occurred at the very same time as EDS. Also, Bassetti and Aldrich (1997) reported that EDS began in the association with prominent psychiatric complaints with parallel fluctuations of mood and EDS in IH patients with a positive family history for a mood disorder.

Some studies agree with the idea that EDS precedes or is concomitant rather than subsequent to mood disorders because non-depressed subjects with a family history of mood disorder commonly have disturbances of REM sleep (Jaussent et al., 2011; Kaplan et al., 2011). Specifically, the shorter REM latency and increased REM sleep density were associated with the development of mood disorder during follow-up (Rao et al., 2009). The view that shortened REM sleep latency could be a specific marker of mood disorders has been frequently debated (Kupfer and Foster, 1972; Coble et al., 1981; Kupfer et al., 1986).

However, this sleep pattern cannot be used to diagnose of mood disorder because of the great variability in depressed patients (Kupfer and Foster, 1972; Lauer et al., 1991).

\section{The possible aetiologic relationship between IH and depression}

We assume there are four possible relationships between $\mathrm{IH}$ and depressive symptoms. 
Firstly, depressive symptoms in $\mathrm{IH}$ patients might be a consequence of a difficultly adapting to the chronic disorder (Bassetti and Aldrich, 1997; Vernet and Arnulf, 2009; Lopez et al., 2017a). Patients with IH often report poor academic and work performances (Bassetti and Aldrich, 1997; Anderson et al., 2007; Avis et al., 2015; Neikrug et al., 2017) and increased incidence of car or work accidents (Bassetti and Aldrich, 1997; Ozaki et al., 2008; Pizza et al., 2015). EDS also affects social and family life and thus decreases quality of life (Ozaki et al., 2008, 2012; Avis et al., 2015; Neikrug et al., 2017; Miglis et al., 2020). This might lead to a depressive mood and to intensification of depressive symptoms or result in a mood disorder later (Lopez et al., 2017a). However, even after the treatment of EDS with stimulants, depression scores may or may not improve, suggesting that the relationship between $\mathrm{IH}$ and depressive symptoms is more complex than one of mere cause and effect.

Secondly, depressive symptoms might be a possible symptom of the pathological process that causes $\mathrm{IH}$. In the study of Vernet and colleagues (2010) half of patients reported a stressful event before the onset of $\mathrm{IH}$, for example the death of a loved one, divorce, a serious illness, the end of the military service or the end of a high level of sport practice. In addition to that, $23 \%$ of them had insomnia and $38 \%$ of them had a major change in sleep habits before the onset of $\mathrm{IH}$ (Vernet et al., 2010). In our opinion the structure of personality, the overall mood and depressive symptoms may contribute to development of $\mathrm{IH}$.

Thirdly, IH and mood disorders might be two disorders that often occur together. Lastly, EDS might be a symptom of depression and the disease that we call IH might be a variant phenotype of depression. Nevertheless, we do consider that this option is not probable because there are $\mathrm{IH}$ subjects without any mood disturbances and there is not an important pathophysiological link suggesting this speculation.

\section{Conclusion}

$\mathrm{IH}$ is frequently connected with depressive symptoms (or mood disorders) yet the etiopathogenetic relationship is unclear. It is indeed possible that the relationship between $\mathrm{IH}$ and depressive symptoms (or mood disorders) may be more complex than just the result of the handicapping disorder. While research concerning this area is ongoing, we ought to make time to listen to $\mathrm{IH}$ patients and improve symptoms at least symptomatically.

\section{References}

Akiskal, H. S., Benazzi, F. (2005) Atypical depression: A variant of bipolar II or a bridge between unipolar and bipolar II? J. Affect. Disord. 84(2-3), 209-217.

American Academy of Sleep Medicine (2005) International Classification of Sleep Disorders, $2^{\text {nd }}$ Ed. American Academy of Sleep Medicine, Darien.

American Academy of Sleep Medicine (2014) International Classification of Sleep Disorders, $3^{\text {rd }}$ Ed. American Academy of Sleep Medicine, Darien.

American Psychiatric Association (2013) Diagnostic and Statistical Manual of Mental Disorders, $5^{\text {th }}$ Ed. American Psychiatric Association, Washington, D. C. 
Anderson, K. N., Pilsworth, S., Sharples, L. D., Smith, I. E., Shneerson, J. M. (2007) Idiopathic hypersomnia: a study of 77 cases. Sleep 30(10), 1274-1281.

Arnulf, I. (2005) Excessive daytime sleepiness in parkinsonism. Sleep Med. Rev. 9(3), 185-200.

Avis, K. T., Shen, J., Weaver, P., Schwebel, D. C. (2015) Psychosocial characteristics of children with central disorders of hypersomnolence versus matched healthy children. J. Clin. Sleep Med. 11(11), 1281-1288.

Barateau, L., Lopez, R., Arnulf, I., Lecendreux, M., Franco, P., Drouot, X., Leu-Semenescu, S., Jaussent, I., Dauvilliers, Y. (2017a) Comorbidity between central disorders of hypersomnolence and immune-based disorders. Neurology 88(1), 93-100.

Barateau, L., Lopez, R., Franchi, J. A., Dauvilliers, Y. (2017b) Hypersomnolence, hypersomnia, and mood disorders. Curr. Psychiatry Rep. 19(2), 13.

Bassetti, C., Aldrich, M. S. (1997) Idiopathic hypersomnia. A series of 42 patients. Brain 120(Pt 8), 1423-1435.

Beck, A. T., Ward, C. H., Mendelson, M., Mock, J., Erbaugh, J. (1961) An inventory for measuring depression. Arch. Gen. Psychiatry 4, 561-571.

Billiard, M. (1996) Idiopathic hypersomnia. Neurol. Clin. 14(3), 573-582.

Billiard, M., Dauvilliers, Y. (2001) Idiopathic hypersomnia. Sleep Med. Rev. 5(5), 349-358.

Billiard, M., Dolenc, L., Aldaz, C., Ondze, B., Besset, A. (1994) Hypersomnia associated with mood disorders: a new perspective. J. Psychosom. Res. 38, 41-47 (Suppl. 1).

Braga, D. M., Prado, G. F., Bichueti, D. B., Oliveira, E. M. (2016) Positive correlation between functional disability, excessive daytime sleepiness, and fatigue in relapsing-remitting multiple sclerosis. Arq. Neuropsiquiatr. 74(6), 433-438.

Coble, P. A., Kupfer, D. J., Shaw, D. H. (1981) Distribution of REM latency in depression. Biol. Psychiatry 16(5), 453-466.

Dauvilliers, Y., Lopez, R., Ohayon, M., Bayard, S. (2013) Hypersomnia and depressive symptoms: Methodological and clinical aspects. BMC Med. 11, 78 .

Dauvilliers, Y., Evangelista, E., Barateau, L., Lopez, R., Chenini, S., Delbos, C., Beziat, S., Jaussent, I. (2019) Measurement of symptoms in idiopathic hypersomnia: The Idiopathic Hypersomnia Severity Scale. Neurology 92(15), e1754-e1762.

Detre, T., Himmelhoch, J., Swartzburg, M., Anderson, C. M., Byck, R., Kupfer, D. J. (1972) Hypersomnia and manic-depressive disease. Am. J. Psychiatry 128(10), 1303-1305.

Dolenc, L., Besset, A., Billiard, M. (1996) Hypersomnia in association with dysthymia in comparison with idiopathic hypersomnia and normal controls. Pflugers Arch. 431, R303-R304 (6 Suppl. 2).

Hamilton, M. (1960) A rating scale for depression. J. Neurol. Neurosurg. Psychiatry 23, 56-62.

Iber, C., Ancoli-Israel, S., Chesson, A. L., Quan, S. (2007) The AASM Manual for the Scoring of Sleep and Associated Events: Rules, Terminology and Technical Specifications. American Academy of Sleep Medicine, Westchester.

Jaussent, I., Bouyer, J., Ancelin, M. L., Akbaraly, T., Peres, K., Ritchie, K., Besset, A., Dauvilliers, Y. (2011) Insomnia and daytime sleepiness are risk factors for depressive symptoms in the elderly. Sleep 34(8), 1103-1110.

Johns, M. W. (1991) A new method for measuring daytime sleepiness: The Epworth sleepiness scale. Sleep 14(6), 540-545.

Kaplan, K. A., Harvey, A. G. (2009) Hypersomnia across mood disorders: a review and synthesis. Sleep Med. Rev. 13(4), 275-285.

Kaplan, K. A., Gruber, J., Eidelman, P., Talbot, L. S., Harvey, A. G. (2011) Hypersomnia in inter-episode bipolar disorder: Does it have prognostic significance? J. Affect. Disord. 132(3), 438-444.

Kim, T., Lee, J. H., Lee, C. S., Yoon, I. Y. (2016) Different fates of excessive daytime sleepiness: Survival analysis for remission. Acta Neurol. Scand. 134(1), 35-41.

Galušková K.; Šonka K. 
Krbkova, L., Stroblova, H., Bednarova, J. (2015) Clinical course and sequelae for tick-borne encephalitis among children in South Moravia (Czech Republic). Eur. J. Pediatr. 174(4), 449-458.

Kupfer, D. J., Foster, F. G. (1972) Interval between onset of sleep and rapid-eye-movement sleep as an indicator of depression. Lancet 2(7779), 684-686.

Kupfer, D. J., Reynolds, C. F. $3^{\text {rd }}$, Grochocinski, V. J., Ulrich, R. F., McEachran, A. (1986) Aspects of short REM latency in affective states: a revisit. Psychiatry Res. 17(1), 49-59.

Lambert, G. W., Eisenhofer, G., Jennings, G. L., Esler, M. D. (1993) Regional homovanillic acid production in humans. Life Sci. 53(1), 63-75.

Lammers, G. J., Bassetti, C. L. A., Dolenc-Groselj, L., Jennum, P. J., Kallweit, U., Khatami, R., Lecendreux, M., Manconi, M., Mayer, G., Partinen, M., Plazzi, G., Reading, P. J., Santamaria, J., Sonka, K., Dauvilliers, Y. (2020) Diagnosis of central disorders of hypersomnolence: A reappraisal by European experts. Sleep Med. Rev. 52, 101306.

Lauer, C. J., Riemann, D., Wiegand, M., Berger, M. (1991) From early to late adulthood. Changes in EEG sleep of depressed patients and healthy volunteers. Biol. Psychiatry 29(10), 979-993.

Lee, J., Na, G., Joo, E. Y., Lee, M., Lee, J. (2017) Clinical and polysomnographic characteristics of excessive daytime sleepiness in children. Sleep Breath. 21(4), 967-974.

Lippert, J., Halfter, H., Heidbreder, A., Rohr, D., Gess, B., Boentert, M., Osada, N., Young, P. (2014) Altered dynamics in the circadian oscillation of clock genes in dermal fibroblasts of patients suffering from idiopathic hypersomnia. PLoS One 9(1), e85255.

Lippert, J., Young, P., Gross, C., Meuth, S. G., Drager, B., Schirmacher, A., Heidbreder, A. (2019) Specific T-cell activation in peripheral blood and cerebrospinal fluid in central disorders of hypersomnolence. Sleep 42(2), zsy223.

Lopez, R., Barateau, L., Evangelista, E., Dauvilliers, Y. (2017a) Depression and hypersomnia: A complex association. Sleep Med. Clin. 12(3), 395-405.

Lopez, R., Doukkali, A., Barateau, L., Evangelista, E., Chenini, S., Jaussent, I., Dauvilliers, Y. (2017b) Test-

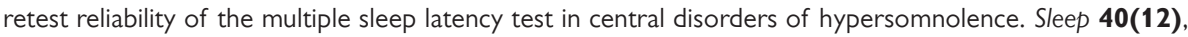
zsx164.

Materna, L., Halfter, H., Heidbreder, A., Boentert, M., Lippert, J., Koch, R., Young, P. (2018) Idiopathic hypersomnia patients revealed longer circadian period length in peripheral skin fibroblasts. Front. Neurol. 9, 424.

Miglis, M. G., Schneider, L., Kim, P., Cheung, J., Trotti, L. M. (2020) Frequency and severity of autonomic symptoms in idiopathic hypersomnia. J. Clin. Sleep Med. 16(5), 749-756.

Montgomery, S. A., Asberg, M. (1979) A new depression scale designed to be sensitive to change. Br. J. Psychiatry 134, 382-389.

Moreno-Lopez, C., Santamaria, J., Salamero, M., Del Sorbo, F., Albanese, A., Pellecchia, M. T., Barone, P., Overeem, S., Bloem, B., Aarden, W., Canesi, M., Antonini, A., Duerr, S., Wenning, G. K., Poewe, W., Rubino, A., Meco, G., Schneider, S. A., Bhatia, K. P., Djaldetti, R., Coelho, M., Sampaio, C., Cochen, V., Hellriegel, H., Deuschl, G., Colosimo, C., Marsili, L., Gasser, T., Tolosa, E. (2011) Excessive daytime sleepiness in multiple system atrophy (SLEEMSA study). Arch. Neurol. 68(2), 223-230.

Neikrug, A. B., Crawford, M. R., Ong, J. C. (2017) Behavioral sleep medicine services for hypersomnia disorders: a survey study. Behav. Sleep Med. 15(2), 158-171.

Ogawa, S., Tsuchimine, S., Kunugi, H. (2018) Cerebrospinal fluid monoamine metabolite concentrations in depressive disorder: A meta-analysis of historic evidence. J. Psychiatr. Res. 105, 137-146.

Ozaki, A., Inoue, Y., Nakajima, T., Hayashida, K., Honda, M., Komada, Y., Takahashi, K. (2008) Health-related quality of life among drug-naive patients with narcolepsy with cataplexy, narcolepsy without cataplexy, and idiopathic hypersomnia without long sleep time. J. Clin. Sleep Med. 4(6), 572-578. 
Ozaki, A., Inoue, Y., Hayashida, K., Nakajima, T., Honda, M., Usui, A., Komada, Y., Kobayashi, M.,

Takahashi, K. (2012) Quality of life in patients with narcolepsy with cataplexy, narcolepsy without cataplexy, and idiopathic hypersomnia without long sleep time: Comparison between patients on psychostimulants, drug-naive patients and the general Japanese population. Sleep Med. 13(2), 200-206.

Pardasani, V., Shukla, G., Singh, S., Goyal, V., Behari, M. (2008) Abnormal sleep-wake cycles in patients with tuberculous meningitis: a case-control study. J. Neurol. Sci. 269(1-2), 126-132.

Pascoe, M., Bena, J., Foldvary-Schaefer, N. (2019) Effects of pharmacotherapy treatment on patient-reported outcomes in a narcolepsy and idiopathic hypersomnia cohort. J. Clin. Sleep Med. 15(12), 1799-1806.

Pizza, F., Jaussent, I., Lopez, R., Pesenti, C., Plazzi, G., Drouot, X., Leu-Semenescu, S., Beziat, S., Arnulf, I., Dauvilliers, Y. (2015) Car crashes and central disorders of hypersomnolence: a French study. PLoS One 10(6), e0129386.

Plante, D. T. (2017) Sleep propensity in psychiatric hypersomnolence: A systematic review and meta-analysis of multiple sleep latency test findings. Sleep Med. Rev. 31, 48-57.

Plante, D. T., Cook, J. D., Goldstein, M. R. (2017) Objective measures of sleep duration and continuity in major depressive disorder with comorbid hypersomnolence: A primary investigation with contiguous systematic review and meta-analysis. J. Sleep Res. 26(3), 255-265.

Pomares, F. B., Boucetta, S., Lachapelle, F., Steffener, J., Montplaisir, J., Cha, J., Kim, H., Dang-Vu, T. T. (2019) Beyond sleepy: Structural and functional changes of the default-mode network in idiopathic hypersomnia. Sleep 42(11), zsz156.

Rao, U., Hammen, C. L., Poland, R. E. (2009) Risk markers for depression in adolescents: Sleep and HPA measures. Neuropsychopharmacology 34(8), 1936-1945.

Roth, B. (1956) Sleep drunkenness and sleep paralysis. Cesk. Neurol. 19(1), 48-58. (in Czech)

Roth, B. (1976) Narcolepsy and hypersomnia: Review and classification of 642 personally observed cases. Schweiz. Arch. Neurol. Neurochir. Psychiatr. 119(1), 31-41.

Roth, B., Nevšímalová, S., Rechtschaffen, A. (1971) Hypersomnia with sleep drunkeness. Cesk. Psychiatr. 67(1), 18-26. (in Czech)

Ruoff, C., Pizza, F., Trotti, L. M., Šonka, K., Vandi, S., Cheung, J., Pinto, S., Einen, M., Simakajornboon, N., Han, F., Peppard, P., Nevšímalová, S., Plazzi, G., Rye, D., Mignot, E. (2018) The MSLT is repeatable in narcolepsy type 1 but not narcolepsy type 2: a retrospective patient study. J. Clin. Sleep Med. 14(1), 65-74.

Rye, D. B., Bliwise, D. L., Parker, K., Trotti, L. M., Saini, P., Fairley, J., Freeman, A., Garcia, P. S., Owens, M. J., Ritchie, J. C., Jenkins, A. (2012) Modulation of vigilance in the primary hypersomnias by endogenous enhancement of GABAA receptors. Sci. Transl. Med. 4(161), 161ra151.

Sforza, E., Hupin, D., Roche, F. (2018) Mononucleosis: A possible cause of idiopathic hypersomnia. Front. Neurol. 9, 922.

Šonka, K., Pazderová, L., Bušková, J., Pretl, M., Dostálová, S., Vorlová, T., Piško, J., Maurovich-Horvath, E., PIchová, L., Volná, J., Př́hodová, I., Suchá, D., Nevšímalová, S., Kemlink, D. (2014) The value of repeated non-confirmatory multiple sleep latency test (MSLT) for the diagnosis of narcolepsy. Cesk. Slov. Neurol. N. 77/110(4), 444-448. (in Czech)

Trotti, L. M., Staab, B. A., Rye, D. B. (2013) Test-retest reliability of the multiple sleep latency test in narcolepsy without cataplexy and idiopathic hypersomnia. J. Clin. Sleep Med. 9(8), 789-795.

Trotti, L. M., Ong, J. C., Plante, D. T., Murray, C. F., King, R., Bliwise, D. L. (2020) Disease symptomatology and response to treatment in people with idiopathic hypersomnia: Initial data from the Hypersomnia Foundation registry. Sleep Med. 75, 343-349.

Vernet, C., Arnulf, I. (2009) Idiopathic hypersomnia with and without long sleep time: A controlled series of 75 patients. Sleep 32(6), 753-759. 
Vernet, C., Leu-Semenescu, S., Buzare, M. A., Arnulf, I. (2010) Subjective symptoms in idiopathic hypersomnia: Beyond excessive sleepiness. J. Sleep Res. 19(4), 525-534.

Zigmond, A. S., Snaith, R. P. (1983) The hospital anxiety and depression scale. Acta Psychiatr. Scand. 67(6), 361-370. 